\section{EVALUATION OF THE EFFICACY AND SAFETY OF AUTOLOGOUS CORD BLOOD TRANSFUSIONS IN VERY LOW-BIRTH-WEIGHT PREMATURE NEWBORNS}

\section{B. Altaş ${ }^{1}$, S. Arsan ${ }^{2}$, E. Okulu², I. Mungan Akin², B. Atasay ${ }^{2}$, S. Kemahli ${ }^{3}$, T. Turmen² \\ ${ }^{1}$ Department of Pediatrics, ${ }^{2}$ Department of Pediatrics, Division of Neonatology, ${ }^{3}$ Department of Pediatrics, Division of Hematology, Ankara University Medical Faculty, Ankara, Turkey}

Background and Aims: To compare the in-vivo efficacy and safety of red cell(RC) concentrates derived from cord blood and banked adult blood in very low-birth-weight premature newborns.

Methods: A prospective, randomized, blindcontrolled study was conducted between March 2008-September 2009. Thirty-nine premature born before $32^{\text {nd }}$ gestation weeks(GW) and/or with birth weight less than $1500 \mathrm{~g}$ were included. RC concentrate bags were prepared from the cord blood, labelled and stored in blood bank. Premature infants requiring blood transfusion were randomly assigned to an autologous or allogeneic product. If an autologous source had been consumed, allogeneic product was used. When patients were discharged, transfusion types were resumed.Two randomized groups were compared on the $14,28,35$ th days and $>35$ days with respect to hemoglobin levels, transfusion numbers, transfusion and phlebotomy volumes, and to hemoglobin, reticulocyte counts and erythropoietin levels in the postnatal 36th and 40th GW.

Results: Among 39 newborns, 16 received autologous transfusion(Group-1), 15 received allogeneic transfusion(Group-2) and 8 did notreceive any transfusion(Group-3). Group-3 had higher birth weight and gestational week $(p<0.05)$, and RDS, clinical sepsis doubt and phlebotomy volumes were less $(p<0.05)$. The reticulocyte counts and erythropoietin levels in the postnatal 36th and 40th GW were lower in Group-1(p<0.05). The other variables did not differ between Group-1 and 2. The autologous source decreased allogeneic transfusion $28 \%$ in Group-1.

Conclusions: Autologous cord blood had the same efficacy and safety with RC concentrates derived from banked adult blood. Careful monitorization of phelobotomy losses, implementing more restrictive transfusion guidelines may increase the efficacy of autologous cord blood.

\section{REGULATORY T-CELLS DIDN'T PLAY A MAJOR ROLE IN THE MAINTENANCE OF SKIN TOLERANCE AFTER PRENATAL MARROW TRANSPLANTATION}

\author{
J.-C. Chen ${ }^{1,2}$, M.-L. Kuo ${ }^{3}$
}

${ }^{1}$ Department of Surgery, Chang Gung Memorial Hospital, ${ }^{2}$ Graduate Institute of Clinical Medical Sciences, College of Medicine, ${ }^{3}$ Department of Microbiology and Immunology, Graduate Institute of Biomedical Sciences, College of Medicine, Chang Gung University, Taoyuan, Taiwan R.O.C.

Background and aims: Reports pertaining to the role of regulatory T-cells (Tregs) in immune tolerance of mixed chimeras had varied greatly, depending upon the protocols or strategies used for the creation of hematopoietic chimerism. We aimed to investigate the requirement of Tregs for tolerance maintenance after in utero marrow transplantation.

Methods: Hematopoietic chimeras were created by in utero marrow transplantation in the gestational days 14 murine fetus. Skin tolerance was defined by the engraftment of donor skin for more than 4 months in the postnatal life. Tregs were quantified by FoxP3 expression using intracellular staining and real-time PCR. In vivo depletion of Tregs was performed by intraperitoneal anti-CD25 injection in mice with skin tolerance.

Results: Tolerant mice had increased Tregs, as evidenced by higher FoxP3 expression among peripheral and splenic CD4 ${ }^{+} \mathrm{T}$-cell populations than non-tolerant and untransplanted mice. Functional assays showed that Tregs from tolerant mice inhibited proliferative alloresponses in a nonspecific manner. However, in vivo depletion of Tregs failed to cause rejection of engrafted donor skins within an observation period of 30 days. Intravenous infusion of $5-7.5 \times 10^{7}$ naïve host lymphocytes caused rapid rejection of engrafted donor skins within 11-19 days as well as failure to accept secondary donor skins transplanted at least 4 months after lymphocyte infusion.

Conclusions: Despite in vivo depletion of increased Tregs in tolerant mice, skin tolerance remained. It argued against a major role of Tregs in the maintenance of skin tolerance following in utero marrow transplantation 